LA W RENCE LIVERMORE N A TIONAL LABORATORY
Partially resolved transition array model for atomic spectra

C. Iglesias, V. Sonnad

December 15, 2011

High energy density physics 
This document was prepared as an account of work sponsored by an agency of the United States government. Neither the United States government nor Lawrence Livermore National Security, LLC, nor any of their employees makes any warranty, expressed or implied, or assumes any legal liability or responsibility for the accuracy, completeness, or usefulness of any information, apparatus, product, or process disclosed, or represents that its use would not infringe privately owned rights. Reference herein to any specific commercial product, process, or service by trade name, trademark, manufacturer, or otherwise does not necessarily constitute or imply its endorsement, recommendation, or favoring by the United States government or Lawrence Livermore National Security, LLC. The views and opinions of authors expressed herein do not necessarily state or reflect those of the United States government or Lawrence Livermore National Security, LLC, and shall not be used for advertising or product endorsement purposes. 


\title{
Partially resolved transition array model for atomic spectra
}

\author{
CARlos A. Iglesias And Vijay Sonnad \\ Lawrence Livermore National Laboratories \\ P.O. Box 808, Livermore, CA 94550, USA
}

\begin{abstract}
The unresolved transition array (UTA) model of atomic spectra describes the lines in a configuration-to-configuration transition array with a single feature that conserves the total strength as well as the energy first and second strength-weighted moments. A new model is proposed that uses a relatively small detailed line calculation together with the extant variance formula to generate a series of Gaussians to describe the transition array. This partially resolved transition array (PRTA) model conserves the known array properties, yields improved higher moments, and systematically accounts for initial level populations. Numerical examples show that the PRTA model provides excellent fidelity to line-by-line methods using only a small fraction of the computational effort for the full calculations.
\end{abstract}

Keywords:

Plasma spectroscopy, transition arrays, opacity, emissivity

Corresponding author:

e-mail: iglesias1@1lnl.gov (C. A. Iglesias) 


\section{Introduction}

An accurate description of plasma radiative properties is important in many research areas including astrophysics and inertial confinement. In cases where the plasma contains partially ionized atoms the detailed line accounting (DLA) method is, in principle, the ideal approach to calculate the bound-bound spectrum.

In local thermal equilibrium (assumed throughout the present work) the spectrum expression using DLA methods is proportional to

$$
\sum_{i} f_{i} g_{i} e^{-\varepsilon_{i} / T} \phi_{i}\left(\omega-\omega_{i}\right)
$$

where the sum is over all spectral lines. Here, $f_{i}, \hbar \omega_{i}$, and $\phi_{i}$ are the oscillator strength, energy, and intrinsic line shape of the $i^{\text {th }}$ line, $\varepsilon_{i}$ and $g_{i}$ are the energy and degeneracy of the initial level for the $i^{\text {th }}$ line, and $T$ is the plasma temperature in energy units. For complex ions, however, the myriad spectral lines make DLA calculations impractical [1]. One approach to circumvent this impasse is the unresolved transition array (UTA) model where the lines in a transition array are treated statistically as a single unresolved feature $[2,3]$.

Consider a transition array consisting of $N$ spectral lines linking two electronic configurations by a dipole radiative transition. The energy-strength moments of the transition array are defined for $q \geq 1$ as

$$
\mu_{q}=S^{-1} \sum_{n=1}^{N}\left(\hbar \omega_{n}\right)^{q} s_{n}
$$

where the sum is over the lines in the transition array and

$$
S=\sum_{n=1}^{N} s_{n}
$$

with $s_{n}$ the strength of the $n^{\text {th }}$ line,

$$
f_{n}=\frac{\hbar \omega_{n}}{3 g_{n}} s_{n} .
$$

Although the moments in Eq. (1.2) can be computed without performing an eigendecomposition of the Hamiltonian, this is computationally expensive (see Appendix). Fortunately, compact formulas are available for the total strength $S$, energy mean $\mu_{1}$, and variance $\sigma=\sqrt{\mu_{2}-\mu_{1}^{2}}$; thus, avoiding the expensive calculations $[2,3]$. 
The implementations of the UTA approach typically choose a Gaussian for the energystrength distribution of the transition array so that the spectral lines are described by

$$
f_{U T A} \exp \left\{-\left(\hbar \omega-\mu_{1}\right)^{2} / 2 \sigma^{2}\right\}
$$

where the array oscillator strength is given by

$$
\begin{aligned}
f_{U T A} & =\frac{\mu_{1}}{3 \Pi} S \\
& \approx \sum_{n=1}^{N} f_{n}
\end{aligned}
$$

with $\Pi$ the degeneracy of the initial configuration. To compute the spectrum the Gaussian in Eq. (1.5) is convolved with the intrinsic line profile and weighted by the abundance of the initial configuration. This procedure allows for a fast calculation of the bound-bound spectrum.

It is known, however, that the Gaussian is often not the proper form of the energy-strength distribution. Indeed, including improved higher moments can yield better agreement with experiments [4,5] and explicit moment calculations show that most transition arrays relevant to Al photon absorption experiments are asymmetrical [1]. Unfortunately, obtaining practical expressions of higher moments for the general case has not been possible [5]. Furthermore, knowledge of higher moments is not sufficient. That is, the true energy-strength distribution is unknown and there are issues with the alternative choices to the Gaussian [5]. Another limitation of the UTA approach is the assumption of statistically populated initial levels, which can impact the spectrum $[1,6]$. Simply using an energy-strength distribution with improved values for the higher moments $\mu_{q}$ does not alleviate this inadequacy (see Appendix).

The DLA methods have two related but separable computational challenges. The first is obtaining the atomic data (line energies and strengths) dominated by the configuration term structure calculation. The second is generating the spectrum from the myriad lines, which can be onerous using straightforward procedures. Recently a method for generating spectra was proposed that is highly efficient for large-scale problems and mitigates the second of these challenges [7].

The purpose here is to present an extension of the UTA approach to complement DLA efforts and reduce the computational time of the atomic data calculation. The proposed model replaces the single feature of a UTA by a number of Gaussians that conserve the known arrays properties and yields improved higher moments. The model also accounts for the dependence on the initial 
level populations. Section 2 introduces the method with numerical examples given in Sections 3 and 4 followed by Conclusions in Section 5.

\section{Partially resolved transition array model}

Assuming there are $M$ open subshells in the transition array, then it can be symbolically written as

$$
\eta_{1}^{v_{1}} \eta_{2}^{v_{2}} \lambda_{3}^{v_{3}} \cdots \lambda_{M}^{v_{M}} \rightarrow \eta_{1}^{v_{1}-1} \eta_{2}^{v_{2}+1} \lambda_{3}^{v_{3}} \cdots \lambda_{M}^{v_{M}}
$$

where $\eta$ and $\lambda$ denote subshell quantum numbers ( $n \ell$ or $n \ell j$ ) and $v$ is the number of electrons occupying the subshell. Here the $\eta^{\prime} s$ represent active subshells in the transition while the $\lambda^{\prime} s$ represent spectator or passive subshells.

The variance of the transition array in Eq. (2.1) is given by [2,3]

$$
\sigma_{t o t}^{2}=\sigma^{2}\left(\eta_{1}^{\nu_{1}} \eta_{2}^{v_{2}} \rightarrow \eta_{1}^{\nu_{1}-1} \eta_{2}^{\nu_{2}+1}\right)+\sum_{m=3}^{M} v_{m}\left(\Pi_{m}-v_{m}\right) \sigma^{2}\left(\lambda_{m} \eta_{1} \rightarrow \lambda_{m} \eta_{2}\right)
$$

where $\Pi_{m}$ is the degeneracy of the $\lambda_{m}$ subshell. The result in Eq. (2.2) has the interesting property that the contributions from each of the passive subshells add separately. Furthermore, since the coefficient in the sum over passive subshells contains all the dependence on the subshell number occupation, the property applies to each spectator electron.

The formula for the variance suggests the following scheme to compute the bound-bound spectra for transition arrays with a large number of lines. The idea is to split the open subshells into two groups: main and secondary. Although this separation is arbitrary, the intent is to include in the main group those electrons that couple strongly to the active electrons while relegating the rest to the secondary group.

\subsection{Detailed line accounting component}

Assume the main group in Eq. (2.1) is defined by the first $M_{K}$ subshells. Now perform a DLA calculation for the transition array that only includes the main group in the angular momentum coupling, but retains the radial integrals for the complete calculation. Symbolically,

$$
\eta_{1}^{v_{1}} \eta_{2}^{v_{2}} \lambda_{3}^{v_{3}} \cdots \lambda_{k}^{v_{k}} \rightarrow \eta_{1}^{v_{1}-1} \eta_{2}^{v_{2}+1} \lambda_{3}^{v_{3}} \cdots \lambda_{M_{K}}^{v_{M_{K}}}
$$

and say it produces $K$ lines.

\subsection{Statistical component}

To conserve the total variance in Eq. (2.2), each of the $K$ lines is assigned the variance, 


$$
\sigma_{K}^{2}=\sum_{m=M_{K}+1}^{M} v_{m}\left(\Pi_{m}-v_{m}\right) \sigma^{2}\left(\lambda_{m} \eta_{1} \rightarrow \lambda_{m} \eta_{2}\right) .
$$

which is the remainder of $\sigma_{t o t}^{2}$ from the secondary group not accounted by the $K$ lines.

\subsection{Model implementation}

The proposed method computes the contribution to the line spectrum in two parts. The first involves an explicit DLA calculation of the main group that is by design small compared to the full DLA calculation. The second portion is statistical and assigns the variance from the secondary group to each of the lines generated in the small-scale DLA calculation. As a result, the method is considered a partially resolved transition array (PRTA) model.

The spectrum for the transition array is described by a sum of Gaussians,

$$
\sum_{k=1}^{K} \bar{f}_{n} \exp \left\{-\hbar^{2}\left(\omega-\bar{\omega}_{k}\right)^{2} / 2 \sigma_{K}^{2}\right\} \bar{g}_{k} e^{-\bar{\varepsilon}_{k} / T}
$$

where the symbols with a bar have the same meaning as in the DLA methods but now for the small-scale DLA calculations. Note that

$$
\sum_{k=1}^{K} \bar{f}_{k} \approx \sum_{n=1}^{N} f_{n}
$$

conserving the total transition array oscillator strength, which depends only on the active subshells except for how the spectator electrons affect line energies [8]. Also, Eq. (2.3.1) includes Boltzmann factors, which approximate the spectrum dependence on initial level populations. As before, the Gaussians are convolved with the intrinsic line profile.

\section{Numerical examples: Single transition array}

The PRTA scheme is now tested against DLA and UTA models. All calculations make the single configuration approximation where the radial integrals are computed in the Dirac-HartreeSlater self-consistent field including Breit and QED corrections [9,10]. The DLA calculations use jj-coupling based on the quasi-spin concept plus the reduced coefficients of fractional parentage [11]. A Voigt profile describes the intrinsic line shape where the Gaussian and Lorentz components are attributed to Doppler and an estimated natural width, respectively. This approximate lower limit of the line width is chosen to minimize merging of the lines in the DLA calculation. All UTA calculations use the spin-orbit split-array version [3]. 
The spectrum has temperature dependence from Doppler broadening and Boltzmann weighting of the initial levels. The temperature in these calculations is chosen so that the ion charge state featured in the example agrees with the ionization average from a Thomas-Fermi [12] calculation at one-hundredth normal density of the material. It is stressed that all the approximate spectra calculations conserve oscillator strength as well as the strength-weighted energy mean and variance of the DLA results.

The examples compare spectra using up to 4 subshells in the main group, which always contain the 2 active subshells. When include, the third and fourth subshells make the largest and next largest contributions to the variance among the passive subshells. Table 1 summarizes the results presenting the number of open subshells and lines in the full and small-scale DLA calculations. The table also gives the fraction of the total variance squared retained in the DLA component of the PRTA scheme,

$$
R \equiv 1-\left(\sigma_{K}^{2} / \sigma_{t o t}^{2}\right)
$$

with $\sigma_{K}^{2}$ and $\sigma_{t o t}^{2}$ given by Eqs. (2.2.1) and (2.2). Finally, Table 1 has estimates of the speedup resulting from the PRTA model relative to the full DLA calculation,

$$
\text { speedup }=\frac{t_{D L A}}{t_{R P T A}}
$$

where $t_{D L A}$ and $t_{R P T A}$ are the times to compute line strengths and positions in the full and smallscale DLA calculations. It is stressed that the speedup does not reflect any timesaving associated with generating the spectrum using a reduced number of lines. This potential savings was excluded since it depends on the number of points in the photon frequency mesh. In some cases the computation time of the small-scale DLA calculation was not large enough to record accurately and was overestimated so the speedup is only a lower bound.

\subsection{Example I}

The first example is the transition array $3 d_{5 / 2}^{2} 4 f_{7 / 2} \rightarrow 3 d_{5 / 2} 4 f_{7 / 2}^{2}$ in $\mathrm{Cd}^{+24}$ with

$$
[M g] 3 p_{1 / 2} 3 p_{3 / 2}^{3} 3 d_{3 / 2}^{2} 3 d_{5 / 2}^{2} 4 s_{1 / 2}^{2} 4 f_{5 / 2} 4 f_{7 / 2}
$$

for initial configuration. The spectra are plotted in Fig. 1 using DLA, UTA, and PRTA with 3 subshells ( $3 p_{3 / 2}^{3}$ plus active subshells) in the main group. The figure shows the PRTA and DLA in good agreement with a large computational savings for the former (see Table 1). On the other hand, the UTA does not capture the sharpness of the array and shoulder on the low energy wing. 


\subsection{Example II}

This example involves an inner shell excitation in $\mathrm{Tm}^{+41}$ with active subshells $2 p_{3 / 2}^{4} 4 d_{5 / 2} \rightarrow 2 p_{3 / 2}^{3} 4 d_{5 / 2}^{2}$ and initial configuration

$$
[M g] 3 p_{1 / 2}^{2} 3 p_{3 / 2}^{2} 3 d_{3 / 2}^{4} 4 s_{1 / 2}^{2} 4 p_{1 / 2} 4 p_{3 / 2} 4 d_{3 / 2} 4 d_{5 / 2} 4 f_{7 / 2}^{2}
$$

The spectra are presented in Fig. 2 and the DLA results show a bimodal feature missed by the UTA approach. On the other hand, the PRTA results with 3 or 4 subshells in the main group $\left(3 p_{3 / 2}^{2}\right.$ and $3 p_{3 / 2}^{2} 4 p_{3 / 2}$ plus active subshells) are in reasonable agreement with the DLA calculation with a speedup of more than three orders of magnitude.

\subsection{Example III}

The last example in this Section considers $3 d_{5 / 2}^{2} \rightarrow 3 d_{5 / 2} 4 p_{1 / 2}$ in $\mathrm{Ni}^{+4}$ for the initial configuration in Eq. (3.1.1). This example has some artificial aspects. Firstly, jj-coupling does not correspond well to the physical conditions [8]. Secondly, such configurations in a weakly ionized atom are probably subject to significant autoionization and configuration interaction effects neglected here [8]. Nevertheless, it tests when the central electron-nuclear interaction is reduced relative to the electron-electron interactions producing the term structure [8].

The results in Fig. 3 show the PRTA calculations with 3 subshells ( $3 p_{1 / 2}$ plus active subshells) in the main group reasonably reproducing the DLA calculations. The UTA spectrum, however, is not in as good agreement with the DLA results. To emphasize the envelope of the line spectrum, DLA calculations with a larger Lorentz width are added to the figure.

\section{Numerical examples: Multiple transition arrays}

Plasma models often consider individual transition arrays accumulating the results to construct the total bound-bound spectrum. To simulate this procedure, examples are provided that add the spectra from many transition arrays. The calculations choose a temperature according to Section 3 and use the same intrinsic profile. Table 2 gives details similar to those in Table 1 . Recall that the speedup only compares the time generating line strengths and positions.

\subsection{Calculations with DLA and UTA models}

For comparisons DLA calculations for all the individual transition arrays are performed as described in Section 3. In addition, separate calculations using the spin-orbit split-array UTA approach [3] for all individual transition arrays were performed. 


\subsection{Calculations with PRTA model}

In the examples the following recipe is used for the PRTA calculations. Firstly, since the primary purpose of the PRTA model is to improve on the UTA approximation whenever the DLA method is deemed impractical, transition arrays with a small number of lines are done explicitly with DLA. Here the limit is set at $10^{4}$ lines for individual transition arrays.

Secondly, the active subshells are always included in the small-scale DLA portion of the calculation. The maximum number of subshells in the main group is limited by

$$
M_{K}=\operatorname{Min}[M-2,4]
$$

where $M$ is the total number of open subshells. For $M_{K} \geq 3$, the first passive subshell included in the main group is the one with the largest contribution to the variance; that is,

$$
v_{3}\left(g_{3}-v_{3}\right) \sigma^{2}\left(\lambda_{3} \eta_{1} \rightarrow \lambda_{3} \eta_{2}\right)=\operatorname{Max}\left\{v_{m}\left(g_{m}-v_{m}\right) \sigma^{2}\left(\lambda_{m} \eta_{1} \rightarrow \lambda_{m} \eta_{2}\right)\right\}_{m=3}^{M}
$$

If a fourth subshell is to be included in the main group it is the subshell with the next largest variance. Note that a fourth subshell is included if

$$
\frac{v_{4}\left(g_{4}-v_{4}\right) \sigma^{2}\left(\lambda_{4} \mu_{1} \rightarrow \lambda_{4} \mu_{2}\right)}{v_{3}\left(g_{3}-v_{3}\right) \sigma^{2}\left(\lambda_{3} \mu_{1} \rightarrow \lambda_{3} \mu_{2}\right)}>0.1
$$

and

$$
\frac{\sigma_{K}^{2}(3 \text { subshells })}{\sigma_{\text {tot }}^{2}}>0.3
$$

otherwise only 3 subshells define the main group.

\subsection{Calculations with mixed DLA-UTA model}

In order to help evaluate the PRTA scheme described in Section 4.2, another model in addition to a all DLA or UTA calculation is considered. Again the DLA method is applied whenever the number of lines in a transition array does not exceed $10^{4}$; otherwise the UTA approach is applied. Except for the choice of cutoff value, such a scheme mimics existing mixed DLA-UTA capabilities [1].

\subsection{Example IV}

The first example in this Section returns to $\mathrm{Cd}^{+24}$ with transitions

$$
3 d^{4} \rightarrow 3 d^{3} 4 f
$$

and initial configuration 
$[M g] 3 p^{4} 3 d^{4} 4 s^{2} 4 f^{2}$

Although the notation is non-relativistic ( $n \ell$ for subshell), jj-coupling is used and all dipole allowed relativistic configurations pairs ( $n \ell j$ for subshell) represented in Eq. (4.4.1) are included in the spectrum. Spectra are compared in Fig. 4 and Table 2 gives details. Although the spectrum using strictly the UTA approach is not in poor agreement with the DLA calculation, the PRTA results show better agreement and significant computational savings relative to the full DLA calculation. On the other hand, the mixed DLA-UTA calculation overlays the UTA result adding a few narrow, weak DLA features.

\subsection{Example $V$}

The final example considers the $\Delta n=0$ transitions $3 p^{4} 3 d^{3} \rightarrow 3 p^{3} 3 d^{4}$ in $\mathrm{Ni}^{+5}$ for the initial configuration

$$
[M g] 3 p^{4} 3 d^{3} 4 s^{2} 4 p^{2}
$$

Similar reservations as Example III apply here. The results in Fig. 5 show the mixed DLA-UTA and PRTA approaches reasonably reproducing the DLA results for the spectrum. Obviously the UTA calculation cannot reproduce the detailed lines. To confirm that the PRTA scheme offers benefits, Fig 6 displays similar calculations using a larger Lorentz width for the intrinsic profile that causes significant line overlap.

The temperature dependence of the spectrum is explored in Fig. 7 where the calculations were repeated at a lower temperature. To emphasize the envelope of the line distribution, the calculations used a larger Lorentz width. The figure shows that the PRTA model, which partially accounts for the initial population induced shift, agrees best with the DLA results.

\section{Conclusions}

A novel partially resolved transition array (PRTA) method was presented that relies on the additive form of the variance formula to split the single feature in the unresolved transition array (UTA) approach into a series of Gaussians while conserving known properties. The PRTA method significantly accelerates detailed line accounting (DLA) calculations and provides better accuracy than models relying on the UTA model.

The PRTA approach separates the open subshells in the transition array into main and secondary groups. The main group is treated with DLA methods to obtain line strengths and energies while the secondary group is treated statistically. The secondary group contributes to the 
spectrum by assigning their portion of the variance, which is computed from existing analytic formulas, to each line from the small-scale DLA calculation. The PRTA conserves the total oscillator strength as well as the strength-weighted energy mean and variance of the transition array. It also improves on the higher energy strength-weighted moments and corrects for initial level population effects in a systematic manner.

The numerical examples show that although the UTA method is in general a reasonable approximation, the PRTA scheme significantly improves agreement with DLA calculations only using a small fraction of the computational effort of the full calculations. The PRTA approach may also prove superior for statistical line-by-line approaches [13] where the individual lines are generated randomly. Such methods require the strength-energy distribution, which is not known and is often approximated by formulas containing free parameters constrained by known exact results. On the other hand, in the PRTA scheme the small-scale DLA calculation gives a good approximation to the energy-strength distribution of the transition array. In addition, only the line splitting produced by the secondary electrons is treated randomly. It is expected that random line treatments based on the PRTA method can account for systematic near coincidences from transition arrays differing only in the distribution of weakly coupled electrons avoiding overestimates of the opacity [14].

The success of the PRTA is readily explained. If the main group contains the strongly coupled electrons to the transition, then the weakly coupled electrons in the secondary group add a relatively small splitting of the lines in the small-scale DLA calculation without significantly redistributing line strengths. The statistical population approximation of the initial levels is also limited to the secondary group and the main temperature dependence contained in the initial level occupations is accounted in the DLA lines. Although the impact of the secondary group on the spectrum is relatively small, it can dramatically increase the computational effort in the DLA calculations. The examples suggest that constructing the main group so that it retains at least $70 \%$ of the variance squared leads to good agreement with DLA calculations.

The recipe defining main and secondary subshells for the PRTA calculations in Section 4 is not intended as universal. It is expected that researchers will vary the computational effort in the small-scale DLA calculation depending on applications and computer resources. Although not attempted in the present work, it is not necessary to include all the electrons in a passive shell in the main group. It is possible to include some of the spectator electrons in a given passive 
subshell leaving other electrons to the secondary group. This segments the variance into smaller increments and could improve the results without significantly increasing the computational effort. Finally, the present numerical examples used jj-coupling, but the concept is obviously applicable to LS or intermediate coupling with suitable modifications.

Acknowledgments: It is pleasure to thank Brian G. Wilson for corrections to the manuscript. This work performed under the auspices of the U.S. Department of Energy by Lawrence Livermore National Laboratory under Contract DE-AC52-07NA27344. 


\section{Appendix}

\section{Calculation of transition array lines and moments}

Consider a dipole transition array linking the initial configuration $C_{b}$ and final configuration $C_{a}$. It is assumed that the two electronic configurations are described by Hamiltonians in distinct atomic subspaces. Consequently, the spectrum at photon energy $\hbar \omega$ can be written in terms of the function [15]

$$
A(\omega)=\pi^{-1} \operatorname{Im}\left[d^{T} \cdot\{(\hbar \omega-i \Gamma) I-L\}^{-1} d\right]
$$

where $\Gamma$ is the natural width,

$$
I=I_{a} \otimes I_{b}
$$

and

$$
L=H_{a} \otimes I_{b}-I_{a} \otimes H_{b} .
$$

Here, $H_{a}$ and $H_{b}$ are the configuration Hamiltonians, $I_{a}$ and $I_{b}$ are identity matrices in the configuration subspaces, $\otimes$ denotes the Kronecker or inner product, the superscript $T$ denotes a transpose, and $u^{T} \cdot w$ denotes a vector dot product. The vector $d$ is defined by

$$
d=\operatorname{vec}(D)
$$

with $D$ the dipole-transition matrix [8] and the vec operation creates a column vector from a matrix by stacking the columns of the matrix below one another.

\section{A.1 Line-by-line calculation}

The standard line-by-line calculation involves and eigendecomposition of the Hamiltonian matrices [8],

$$
Q_{c} H_{c}=E_{c} Q_{c}
$$

where $c=a$ or $b, E_{c}=\operatorname{diag}\left\{\varepsilon_{c 1}, \varepsilon_{c 2}, \ldots\right\}$ with $\varepsilon_{c}$ the eigenvalues of $H_{c}$, and the columns of the matrix $Q_{c}$ are the eigenvectors. Since $H_{c}$ is real and symmetric (Hermitian), the $\varepsilon_{c}{ }^{\prime} s$ are real and $Q_{c}$ is a similarity transformation; that is, $Q_{c} Q_{c}^{T}=Q_{c}^{T} Q_{c}=I_{c}$.

Applying the eigendecomposition in Eq. (A.1.1) to the function in Eq. (A.1); that is, use

$$
\left(Q_{a} \otimes Q_{b}\right) L=\left[E_{a} \otimes I_{b}-I_{a} \otimes E_{b}\right]\left(Q_{a} \otimes Q_{b}\right)
$$

to get

$$
\operatorname{Lim}_{\Gamma \rightarrow 0} A(\omega)=\hbar^{-1} \sum_{n=1}^{N} s_{n} \delta\left(\omega-\omega_{n}\right)
$$


where the sum is over all lines of the transition array. The strength and energy of the $n^{\text {th }}$ line are

$$
\begin{aligned}
& s_{n}=\left\{n^{\text {th }} \text { element of }\left[\operatorname{vec}\left(Q_{b}^{T} D Q_{a}\right)\right]\right\}^{2}, \\
& \hbar \omega_{n}=n^{\text {th }} \text { eigenvalue of }\left[E_{a} \otimes I_{b}-I_{a} \otimes E_{b}\right],
\end{aligned}
$$

which agree with the standard atomic data calculations [8].

\section{A.2 Moment calculation}

The frequency moments of the function $A(\omega)$ are related to its expansion in powers of the operator $L[16]$,

$$
\begin{aligned}
M_{q} & =\int_{-\infty}^{\infty} d \omega \omega^{q}\left\{\operatorname{Lim}_{\Gamma \rightarrow 0} A(\omega)\right\} \\
& =d^{T} \cdot(L / \hbar)^{q} d
\end{aligned}
$$

Using the eigendecomposition in Eq. (A.1.2) together with the formula

$$
(B \otimes A) \operatorname{vec}(D)=\operatorname{vec}\left(A D B^{T}\right)
$$

it can be readily shown that the energy moments in Eq. (1.1) can be expressed as

$$
\mu_{q}=S^{-1} \hbar^{q} M_{q}
$$

Furthermore, repeated use of Eq. (A.2.2) yields for the frequency moments

$$
M_{q}=\operatorname{vec}(D)^{T} \cdot \sum_{k=0}^{q} \frac{(-1)^{q} q !}{k !(q-k) !} \operatorname{vec}\left(H_{b}^{q} D H_{a}^{q-k}\right)
$$

Consequently, the calculation of the transition array strength-energy moments can be performed by constructing the Hamiltonian and dipole-transition matrices, but does not require the eigendecomposition of the Hamiltonian matrices as implied by Eq. (1.1).

\section{A.3 Operation count}

For simplicity assume that the matrices $H_{a}$ and $H_{b}$ cannot be further reduced into diagonal blocks (if $H_{a}$ and $H_{b}$ represent a diagonal block of the total Hamiltonian, then the total spectrum is obtained by summing over all such blocks). Thus, $H_{a}$ and $H_{b}$ are square matrices of size $n_{a} \times n_{a}$ and $n_{b} \times n_{b}$, respectively, $D$ is rectangular of size $n_{b} \times n_{a}$, which generates $N=n_{a} n_{b}$ spectral lines. Also assume that $n_{a} \approx n_{b} \approx n$. 
The operation count to obtain the line strengths and energies is given by the eigendecomposition of two real symmetric matrices plus the two matrix multiplications in Eq. (A.1.4a) and is therefore [17]

$$
C_{D L A} \approx 19 n^{3}+O\left(n^{2}\right)
$$

The operation count to obtain $\left\{M_{1}, \cdots, M_{q}\right\}$ using Eq. (A.2.4) is

$$
C_{q} \approx 2 q n^{3}+O\left(n^{2}\right)
$$

(care is necessary not to double count some operations giving $q^{2}$ for the coefficient). Ignoring possible acceleration from optimized subroutines (e.g.; matrix multiply), a comparison of the operation counts shows that computing the moments exceeds the cost of the DLA results at $q \approx 10$. More importantly, the cost of calculating each moment is $O\left(n^{3}\right)$, which is impractical for large-scale transition arrays.

\section{A.4 Initial populations}

Following the approximations in the UTA approach [2,3], Eq. (A.1) assumes statistically populated initial levels. To include initial level populations explicitly, replace the second dipole vector in Eq. (A.1) by [15]

$$
d \rightarrow \operatorname{vec}(\rho D)
$$

where $\rho$ is the density matrix for the initial configuration, which in local thermal equilibrium is a functional of $H_{b}$. The result in Eq. (A.2.4) now becomes

$$
M_{q} \rightarrow \operatorname{vec}(D)^{T} \cdot \sum_{k=0}^{q} \frac{(-1)^{q} q !}{k !(q-k) !} \operatorname{vec}\left(H_{b}^{q} \rho D H_{a}^{q-k}\right)
$$

and is considerably more difficult to evaluate without the spectrum of $H_{b}$ than Eq. (A.2.4). 


\section{References}

[1] Q. Pocheron, J.-Ch. Pain, F. Gilleron, T. Blenski, HEDP 7, 234(2011)

[2] C. Bauche-Arnoult, J. Bauche \& M. Klapisch, Phys.Rev. A20, 2424(1979)

[3] C. Bauche-Arnoult, J. Bauche \& M. Klapisch, Phys.Rev. A31, 2248(1985)

[4] F. Gilleron, J.-Ch. Pain, J. Bauche \& C. Bauche-Arnoult, Phys.Rev. E77, 026708(2008)

[5] J.-Ch. Pain, F. Gilleron, J. Bauche \& C. Bauche-Arnoult, HEDP 5, 294(2009)

[6] F. Gilleron, J.-Ch. Pain, Q. Pocherot, J. Bauche \& C. Bauche-Arnoult, HEDP. 7, 277(2011)

[7] V. Sonnad \& C.A. Iglesias, HEDP 7, 43(2011)

[8] R. Cowan, The Theory of Atomic Structure (California Press, Berkeley, 1974)

[9] D.A. Liberman, Phys.Rev. B2, 244(1970)

[10] K.N. Huang et al., At. Data Nucl. Data Tables 18, 243(1976)

[11] G. Gaigalas, S. Fritzsche \& I.P. Grant, Comput. Phys. Commun. 139, 263(2001)

[12] R.P. Feyman, N Metropolis \& E. Teller, Phys.Rev. 75, 1561(1949)

[13] F. Gilleron, J. Bauche \& C. Bauche-Arnoult, J.Phys. B. 40, 3057(2007)

[14] C.A. Iglesias \& B.G. Wilson, JQSRT 52, 127(1994)

[15] H.R. Griem, Spectral Line Broadening by Plasma (Academic, new York, 1974)

[16] D.A. McQuarrie, Statistical Mechanics (Harper \& Row, New York, NY, 1976)

[17] J.W. Demmel, Applied Numerical Linear Algebra, (Society for Industrial and Applied Mathematics, 1997) 
TABle 1

Model comparison for single arrays

\begin{tabular}{|c|c|c|c|c|c|c|}
\hline & \multicolumn{2}{|c|}{ DLA } & \multicolumn{4}{c|}{ PRTA } \\
\hline Example & $M^{a}$ & $N^{b}$ & $M_{K}^{a}$ & $K^{b}$ & $R$ & Speedup \\
\hline I & 6 & $4,499,474$ & 3 & 1351 & 0.88 & $>500^{\mathrm{c}}$ \\
\hline \multirow{2}{*}{ II } & 7 & $11,437,332$ & 4 & 1829 & 0.99 & $>5000^{\mathrm{c}}$ \\
\cline { 4 - 7 } & & & 3 & 145 & 0.83 & $>5000^{\mathrm{c}}$ \\
\hline III & 7 & $4,708,759$ & 3 & 10 & 0.85 & $>750^{\mathrm{c}}$ \\
\hline
\end{tabular}

${ }^{a}$ Number of open subshells in the full or small-scale DLA calculation.

${ }^{b}$ Number of lines in the full or small-scale DLA calculation.

${ }^{\mathrm{c}}$ Calculation times for the small-scale DLA are too small to determine accurately.

TABle 2

Model comparison for multiple arrays

\begin{tabular}{|c|c|c|c|c|c|c|}
\hline & DLA & UTA & Mixed DLA-UTA & \multicolumn{2}{|c|}{ PRTA } \\
\hline Example & $\sum N^{a}$ & $N_{U T A}^{b}$ & $\sum N^{a}$ & $N_{U T A}^{b}$ & $\sum K^{a}$ & Speedup \\
\hline IV & $74,958,755$ & 108 & 82,059 & 81 & 235,308 & 225 \\
\hline $\mathbf{V}$ & $1,936,718$ & 96 & 118,973 & 31 & 137,993 & 10 \\
\hline
\end{tabular}

a Total number of lines in the full, mixed, or small-scale DLA calculation.

${ }^{\mathrm{b}}$ Number of transition arrays treated with UTA approach. 


\section{Figure Captions}

Fig. 1 Spectrum calculations for Example I with $T=150 \mathrm{eV}$ and $\Gamma=0.02 \mathrm{eV}$ : DLA (solid black), UTA (dash black), PRTA with 3 subshells in the main group (red).

Fig. 2 Spectrum calculations for Example II with $T=360 \mathrm{eV}$ and $\Gamma=0.7 \mathrm{eV}$ : DLA (solid black), UTA (dash black), PRTA with 3 (red) and 4 (green) subshells in the main group.

Fig. 3 Spectrum calculations for Example III with $T=11 \mathrm{eV}$ and $\Gamma=0.004 \mathrm{eV}$ : DLA (solid black), UTA (dash black), PRTA with 3 subshells in the main group (red). Also, DLA calculation with $\Gamma=0.3 \mathrm{eV}$ (green).

Fig. 4 Spectrum results for Example IV with $T=150 \mathrm{eV}$ and $\Gamma=0.02 \mathrm{eV}$ : DLA (solid black), UTA (dash black), mixed DLA-UTA (blue), and PRTA (red).

Fig. 5 Spectrum results for Example $\mathrm{V}$ with $T=20 \mathrm{eV}$ and $\Gamma=0.001 \mathrm{eV}$ : DLA (solid black), UTA (dash), mixed DLA-UTA (blue), and PRTA (red).

Fig. 6 Same as Fig. 5 with $\Gamma=0.3 \mathrm{eV}$.

Fig. 7 Same as Fig. 5 with $T=5 e V$ and $\Gamma=1 e V$. 


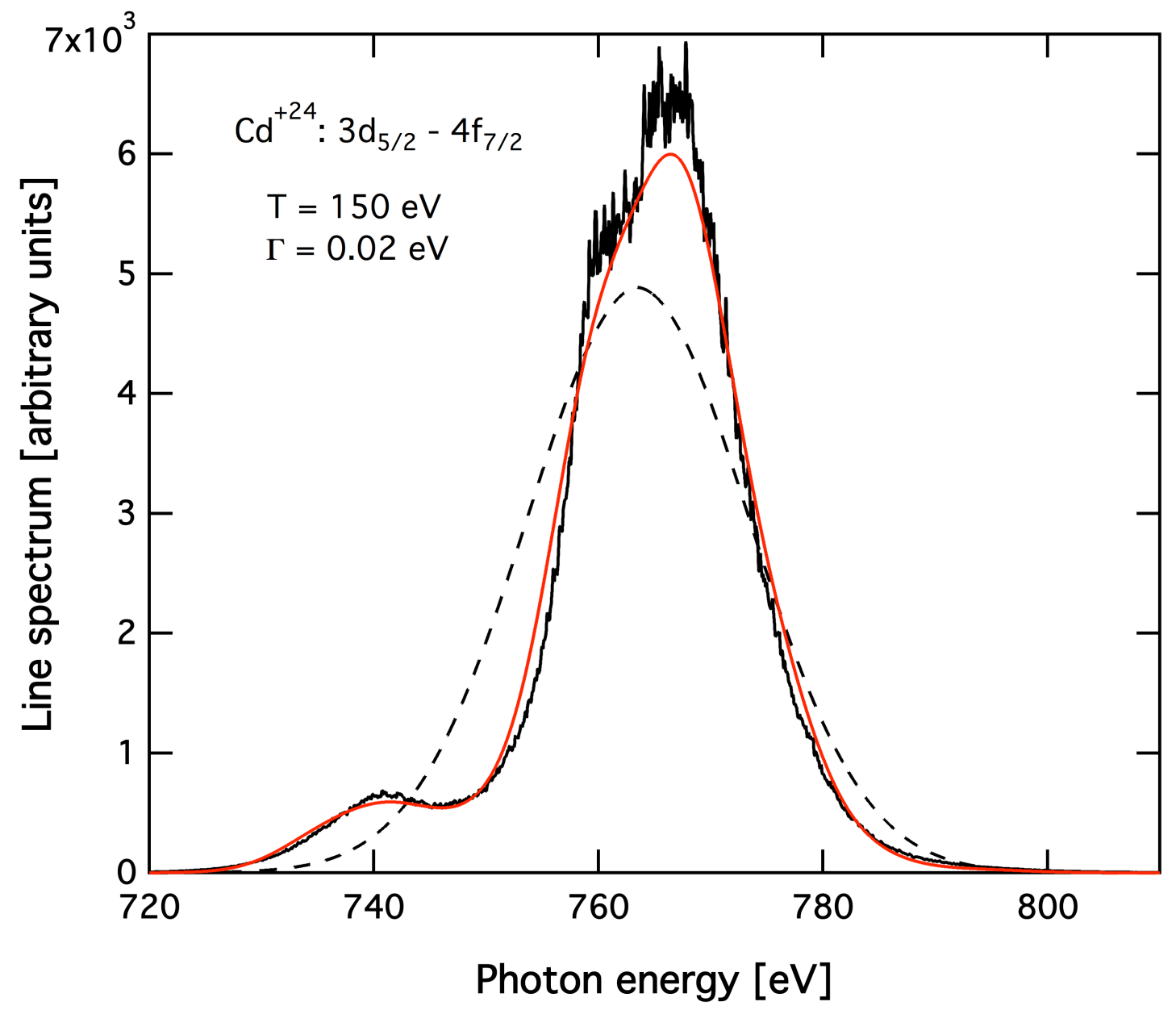

Fig. 1 Spectrum calculations for Example I with $T=150 \mathrm{eV}$ and $\Gamma=0.02 \mathrm{eV}$ : DLA (solid black), UTA (dash black), PRTA with 3 subshells in the main group (red). 


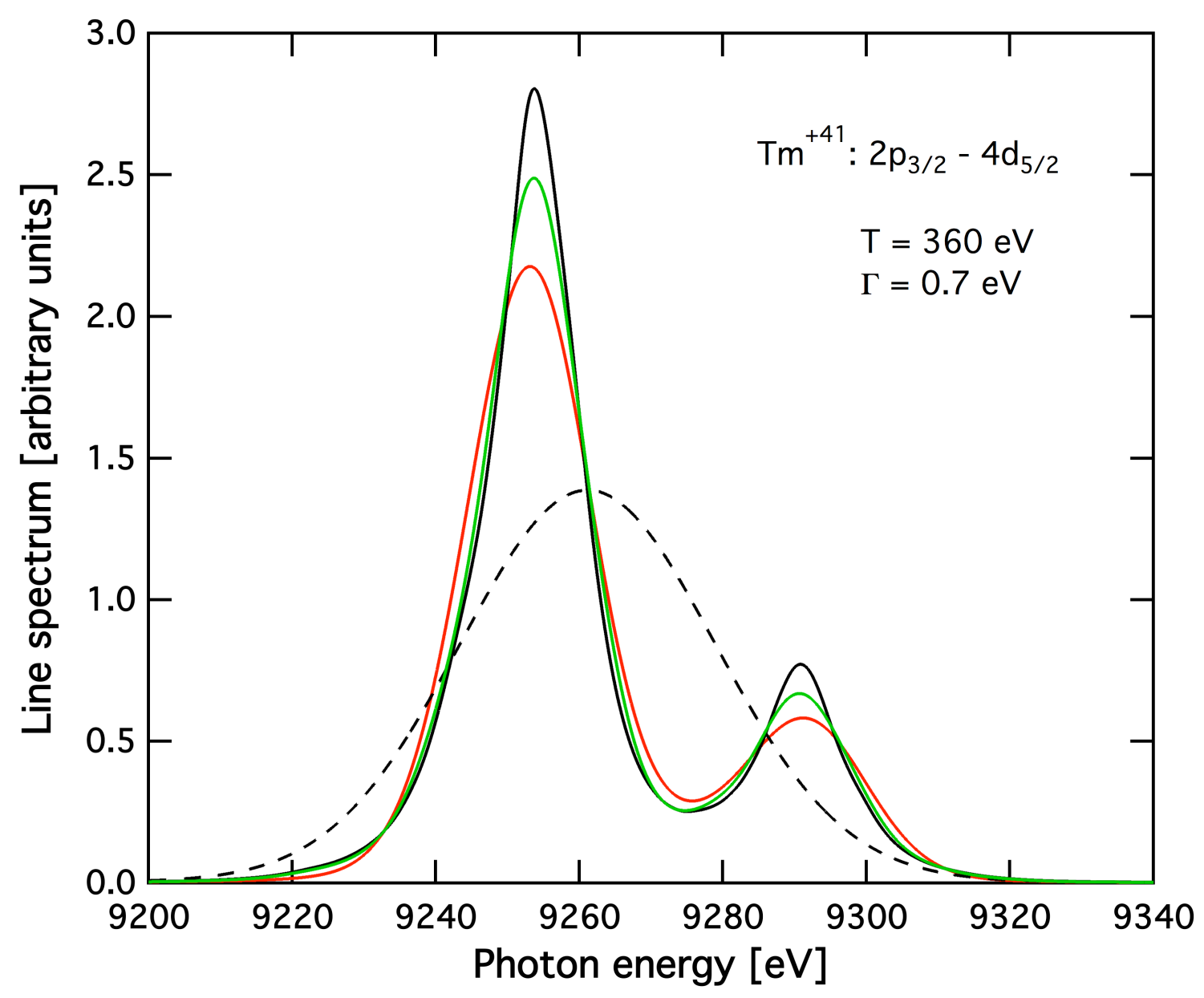

Fig. 2 Spectrum calculations for Example II with $T=360 \mathrm{eV}$ and $\Gamma=0.7 \mathrm{eV}$ : DLA (solid black), UTA (dash black), PRTA with 3 (red) and 4 (green) subshells in the main group. 


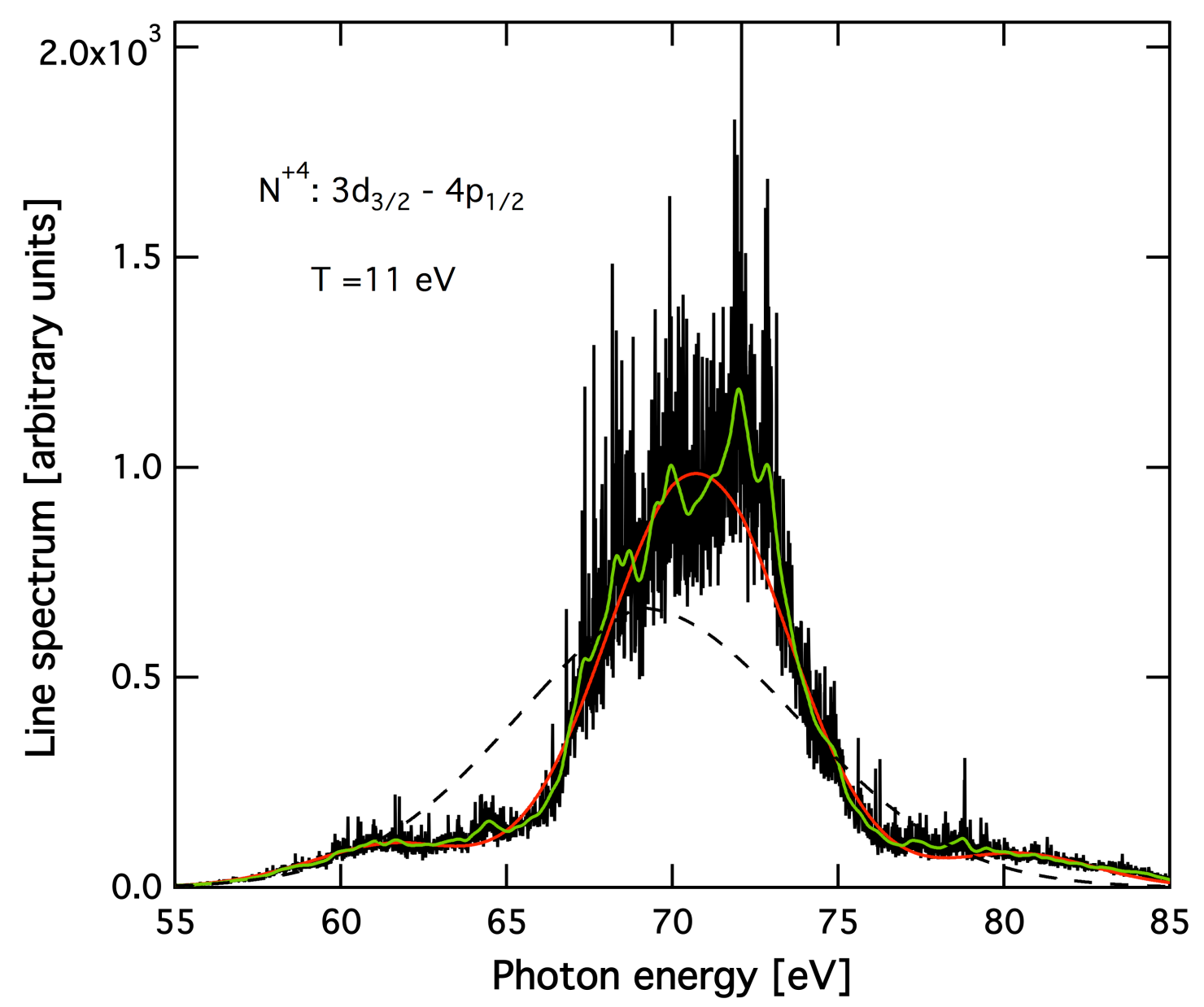

Fig. 3 Spectrum calculations for Example III with $T=11 \mathrm{eV}$ and $\Gamma=0.004 \mathrm{eV}$ : DLA (solid black), UTA (dash black), PRTA with 3 subshells in the main group (red). Also, DLA calculation with $\Gamma=0.3 \mathrm{eV}$ (green). 


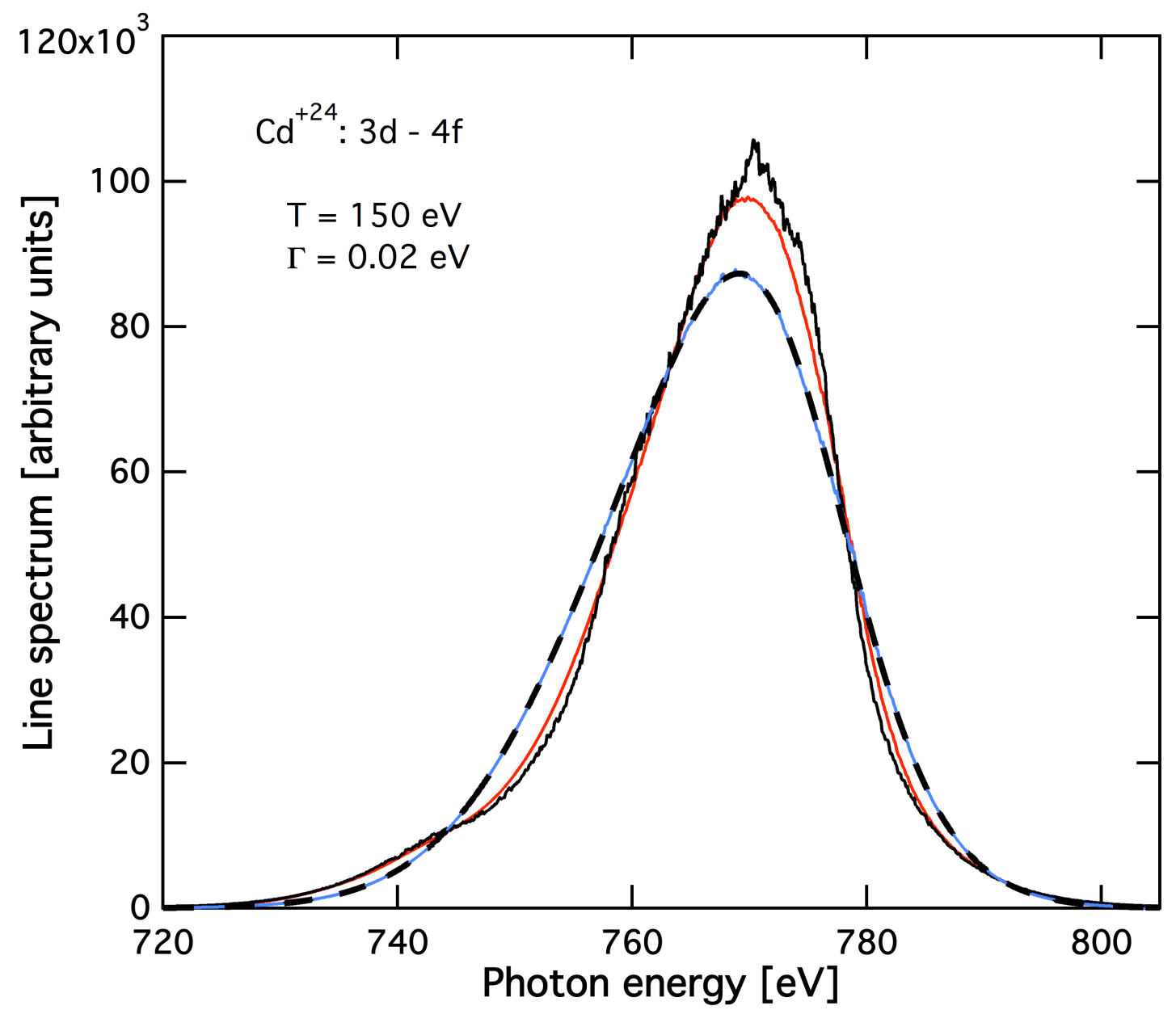

Fig. 4 Spectrum results for Example IV with $T=150 \mathrm{eV}$ and $\Gamma=0.02 \mathrm{eV}$ : DLA (solid black), UTA (dash black), mixed DLA-UTA (blue), and PRTA (red). 


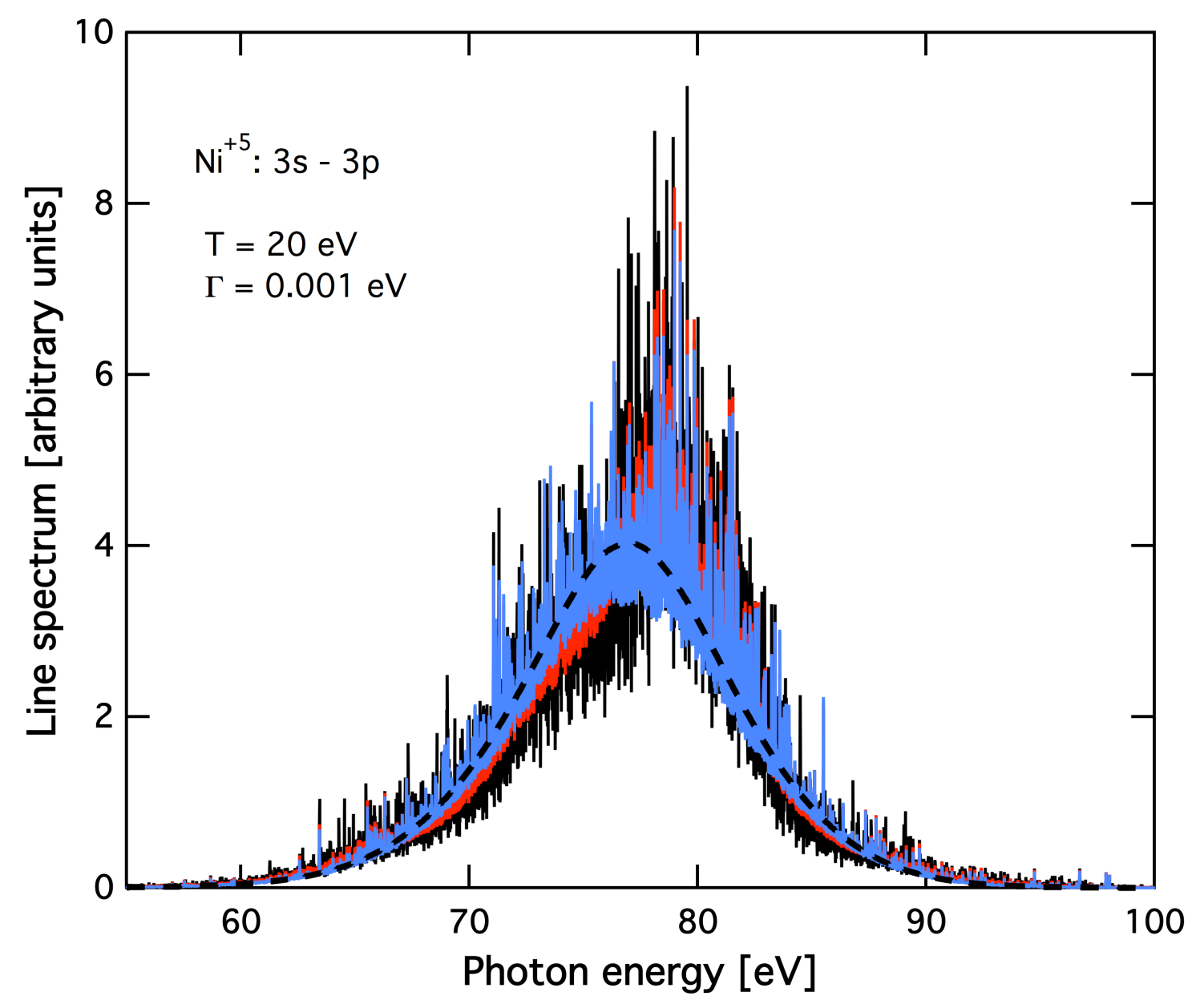

Fig. 5 Spectrum results for Example $\mathrm{V}$ with $T=20 \mathrm{eV}$ and $\Gamma=0.001 \mathrm{eV}$ : DLA (solid black), UTA (dash), mixed DLA-UTA (blue), and PRTA (red). 


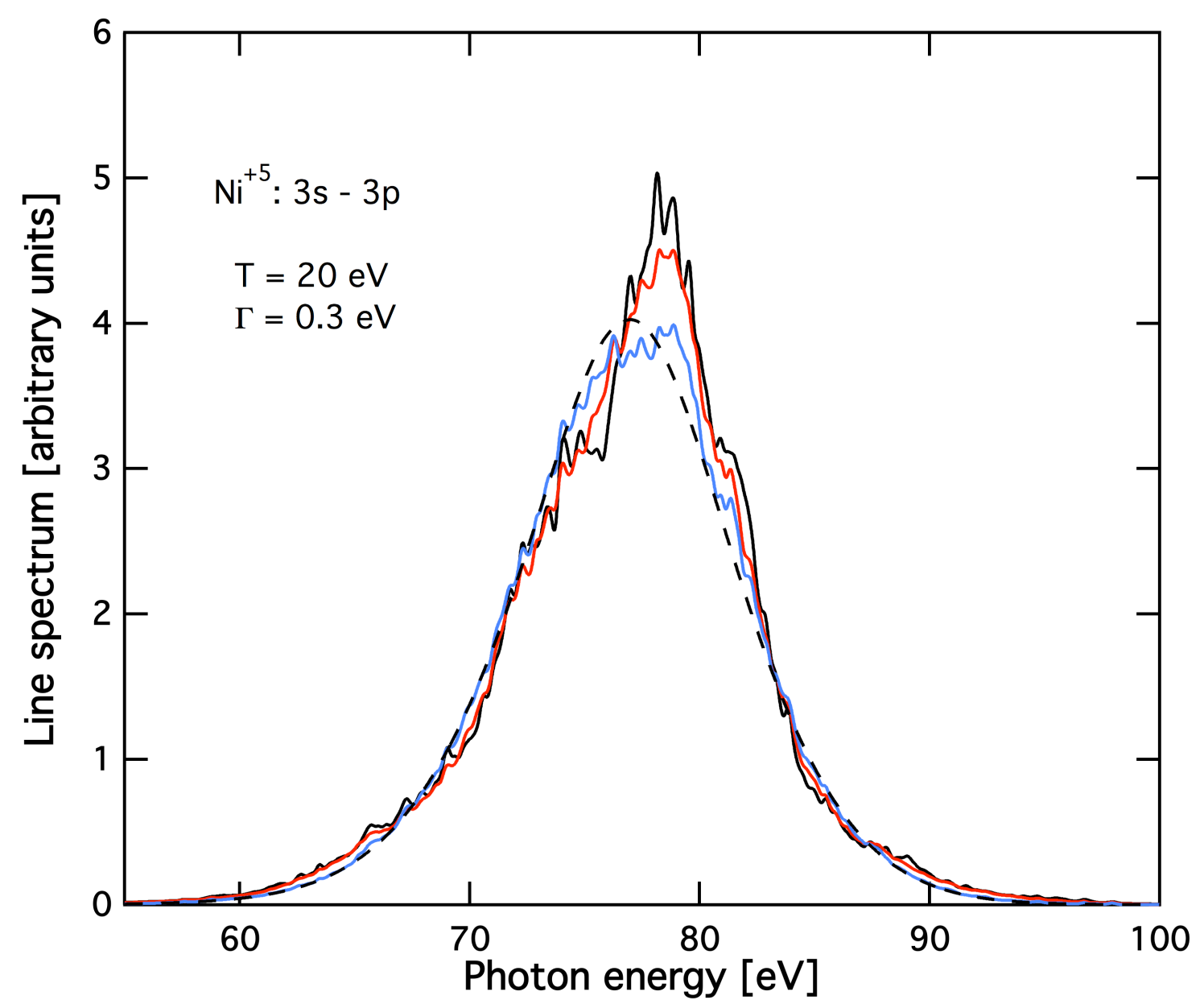

Fig. 6 Same as Fig. 5 with $\Gamma=0.3 \mathrm{eV}$. 


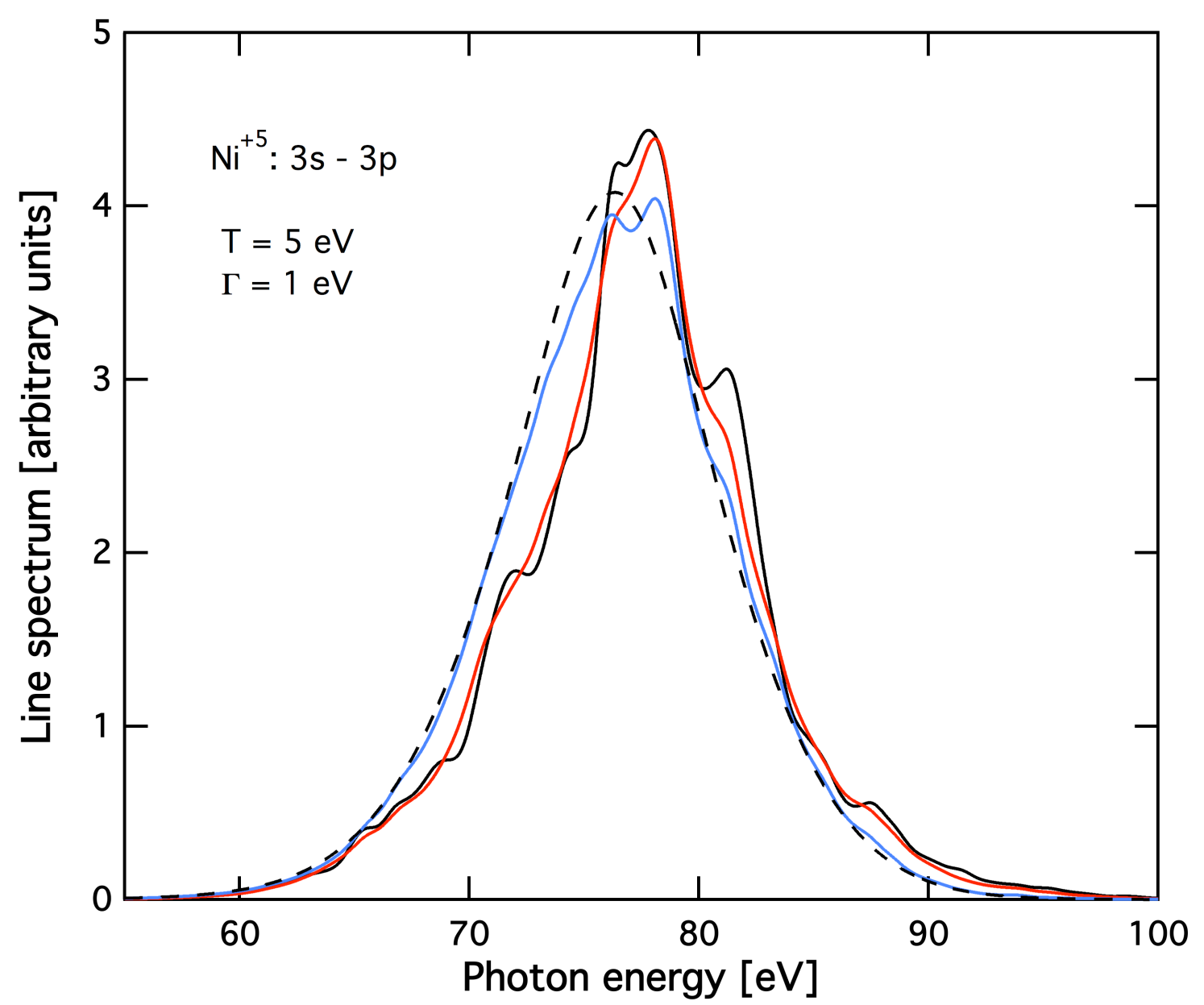

Fig. 7 Same as Fig. 5 with $T=5 e V$ and $\Gamma=1 \mathrm{eV}$. 